\title{
One-Dimensional Single- and Dual-Cavity Magnetophotonic Crystal Fabricated by Bonding
}

\author{
Yoji Haga, Taichi Goto, Alexander V. Baryshev", **, and Mitsuteru Inoue \\ Department of Electrical and Electronic Information Engineering, Toyohashi Tech., Toyohashi, Aichi 441-8580, Japan \\ ${ }^{*}$ Electronics-Inspired Interdisciplinary Research Institute (EIIRIS), Toyohashi Tech., Toyohashi, Aichi 441-8580, Japan \\ ${ }^{* *}$ Ioffe Physico-Technical Institute, St. Petersburg 194021, Russia
}

Dual-cavity magnetophotonic crystals (MPCs) based on bismuth-substituted yttrium iron garnet (Bi:YIG) have been fabricated by a bonding method, and their structural, optical and magneto-optical properties have been studied. Identical structure of bonded fractions revealed the existence of the Fabri-Perot resonances in transmission spectra of single- and dual-cavity MPCs and corresponding enhancement of the Faraday rotation. However, the bonding interface was found to be unstable because of the bonding surfaces' roughness introduced by crystallization of Bi:YIG.

Key words: magnetophotonic crystal, Faraday rotation, bonding, single cavity, dual cavity

\section{Introduction}

Theoretical considerations of magnetophotonic crystals (MPCs) have resulted in concepts of enhancement of polarization rotation for light traveling in MPCs ${ }^{1)}$, the tunable photonic band structure ${ }^{2)}$, the magnetic superprism ${ }^{3)}$, the optical isolator ${ }^{4,5)}$ and circulator ${ }^{6}$. Among these considerations is a concept of dual cavities or one-dimensional (1D) MPCs containing two defects. Works ${ }^{5,7,8)}$ report that such dual-cavities can be optimized so that they exhibit large transmissivity and the Faraday rotation angle up to $45^{\circ}$. From application point of view, MPCs exhibiting large magneto-optical (MO) responses and fast switching speed are attractive for several applications $1,9,10$ ).

The phenomenon of the Faraday rotation originates from the difference in the refractive index for right- and left-circularly polarized (LC-and RC-polarized) waves traveling in a magnetized medium. Analysis of MPCs having the micro-cavity structure shows that the Faraday rotation rises as the thickness of Bragg mirrors increases. This happens due to a strong light localization in the MO defect (Fabri-Perot resonance) and the nonreciprocity of the Faraday effect, accumulating the polarization rotation for light bouncing in the MO defect. However, the full width at half maximum (FWHM) of the Fabri-Perot resonance decreases with the thickness of the Bragg mirrors. And, considering circular polarization-resolved spectra of MPCs, the LC-and RC-polarized resonances split because of difference in the refractive index of the MO defect for the LC and RC modes. At the condition that FWHM is narrower than this split, transmittance of single-cavity MPCs will be substantially lower.

To solve this problem, Steel et. al. ${ }^{11)}$ considered theoretically the MPCs with two defects (the dual cavities). Such MPCs exhibit transmission up to $100 \%$ transmissivity and the Faraday rotation angle of $45^{\circ}$. However, the suggested structure comprises a number of magnetic layers. From a view point of present fabrication possibilities, high-quality MPCs with such designs are unrealizable. That is why Inoue et. al. $\left.{ }^{8}\right)$ simplified the design of MO dual cavities, where two MO defects were symmetrically introduced between three Bragg mirrors. Their studies showed that such MPCs have lower optical and MO responses as compared with that reported in Ref. 12).

Most of realized MPCs are based on bismuth-substituted yttrium iron garnet (Bi:YIG) ${ }^{13)}$ which is one of the best MO materials with a relatively high transmissivity in the visible spectral range. However, sputtered Bi:YIG layers require annealing for their crystallization. This annealing being accompanied by crystallization of $\mathrm{Bi}$ :YIG and $\mathrm{Ta}_{2} \mathrm{O}_{5}$ worsens optical properties of the Bragg mirrors. It has been shown that dual-cavity MPCs fabricated by successive sputtering with double annealing Bi:YIG layers have poor optical properties ${ }^{12)}$. Deviation thicknesses from the designed and non-ideal interfaces between layers are the limiting factors.

The present work is aimed at how to overcome the aforementioned problem. We have demonstrated that the bonding method is feasible for fabricating dual-cavity MPCs shown in Fig. 1. Fabrication steps were as follows: (i) preparation of a single-cavity MPC on a one-inch substrate by sputtering, (ii) dividing the

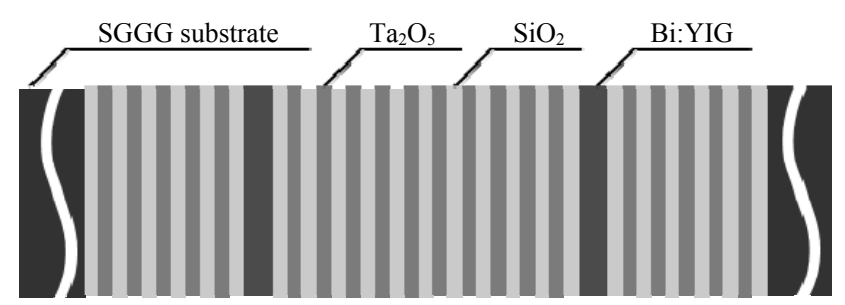

Fig. 1 Sketch of a magnetophotonic dual microcavity with two magnetic defects. 
single cavity in half, and (iii) bonding these half-inch fractions. Since the one-half fractions have identical structural properties, the dual-cavity MPCs exhibited better responses than that reported previously ${ }^{12}$.

\section{Bonding for non-magnetic photonic crystals}

The chemical wet bonding process was found to be suitable for bonding one-dimensional photonic crystals (PCs). Surfaces of sputtered Bragg mirrors, in which the last layer was $\mathrm{SiO}_{2}$ one, were modified in order to obtain highly hydrophilic surfaces. This was done by dipping half-inch samples into a chemical solution of $\mathrm{H}_{2} \mathrm{SO}_{4}: \mathrm{H}_{2} \mathrm{O}_{2}$. Particular treatment was as follows. Samples were cleaned by ultrasonic waves in 2-propanol and then in acetone. These samples were kept in $\mathrm{H}_{2} \mathrm{SO}_{4}: \mathrm{H}_{2} \mathrm{O}_{2}$ at $80{ }^{\circ} \mathrm{C}$ for 1 hour and, finally, were pressed to each other by tweezers (Fig. 2).

Bonded single cavities had $\mathrm{Q} /(\mathrm{T} / \mathrm{S})^{5}-(\mathrm{S} / \mathrm{T})^{5 / Q}$ structure, where $\mathrm{Q}$ was a synthetic fused quartz substrate, $\mathrm{T}$ was a $\lambda / 4$-thick $\mathrm{Ta}_{2} \mathrm{O}_{5}$ layer, and a $\lambda / 4$-thick $\mathrm{S}$ was $\mathrm{SiO}_{2}$ layer. Figure 3 shows transmission spectra of the $(\mathrm{S} / \mathrm{T})^{5} / \mathrm{Q}$ and $\mathrm{Q} /(\mathrm{T} / \mathrm{S})^{5}-(\mathrm{S} / \mathrm{T})^{5} / \mathrm{Q}$ samples. One can see a photonic band originated from the Bragg diffraction and a Fabri-Perot peak due to existence of resonant light coupling to the $\lambda / 2$-thick $\mathrm{SiO}_{2}$ defect layer. Provided that the stop band center wavelength for the

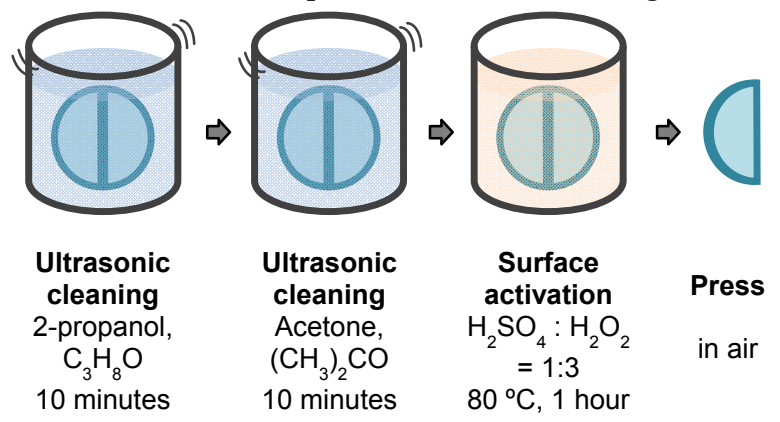

Fig. 2 Bonding procedure with surface activation by wet etching.

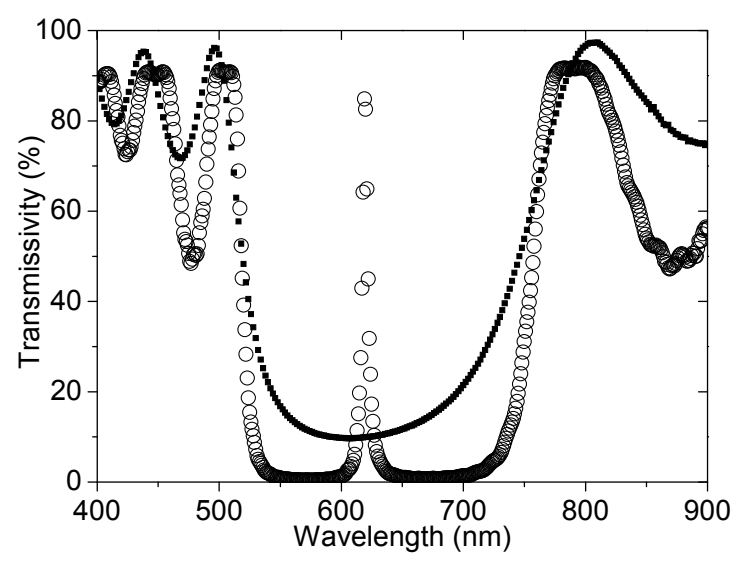

Fig. 3 Transmission spectra of a bonded single-cavity PC with a structure of $\mathrm{Q} /(\mathrm{T} / \mathrm{S})^{5}-(\mathrm{S} / \mathrm{T})^{5} / \mathrm{Q}$ (open circles) and an initial $\mathrm{Q} /(\mathrm{T} / \mathrm{S})^{5}$ multilayer (solid squares).

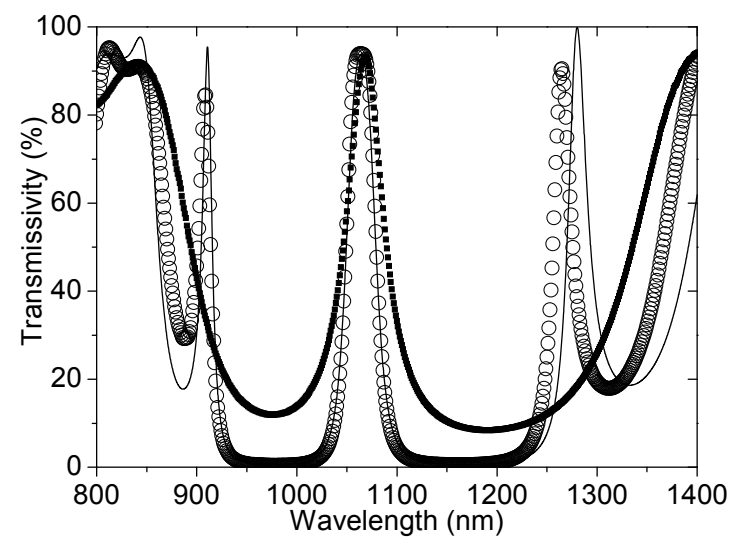

Fig. 4 Transmission spectra of a bonded dual-cavity $\mathrm{PC}$ with a structure of $\mathrm{Q} /(\mathrm{T} / \mathrm{S})^{3 / T} /(\mathrm{S} / \mathrm{T})^{3} / \mathrm{S}-\mathrm{S} /(\mathrm{T} / \mathrm{S})^{3 / T} /(\mathrm{S} / \mathrm{T})^{3 / 2} \mathrm{Q}$

(experiment-open circles, calculation-line) and that of an initial $\mathrm{Q} /(\mathrm{T} / \mathrm{S})^{3 / \mathrm{T}} /(\mathrm{S} / \mathrm{T})^{3}$ single cavity (solid squares).

Bragg mirrors was $613 \mathrm{~nm}$ and the spectral position of the resonance was $619 \mathrm{~nm}$, one may state that bonding was successful and that there was not an air gap between the bonded fractions.

Figure 4 presents spectra of single-cavity and dual-cavity PCs fabricated along the bonding procedure discussed above. Single cavities had a structure of $\mathrm{Q} /(\mathrm{T} / \mathrm{S})^{3} / \mathrm{T} /(\mathrm{S} / \mathrm{T})^{3}$ characterized by with the resonant wavelength of $1064 \mathrm{~nm}$. The bonded dual-cavity MPC had a structure of $\mathrm{Q} /(\mathrm{T} / \mathrm{S})^{3} / \mathrm{T} /(\mathrm{S} / \mathrm{T})^{3 / \mathrm{S}}-\mathrm{S} /(\mathrm{T} / \mathrm{S})^{3 / T} /(\mathrm{S} / \mathrm{T})^{3 / \mathrm{Q}} ; \quad$ the bonded interfaces were the $\mathrm{SiO}_{2}$ layers. One can see that the peaks of resonant transmission coincided for the cavities under study. The experimental (symbol) and calculated (gray line) transmission spectra of the dual-cavity MPC are in good agreement. One can conclude that bonding process is useful for fabrication of the thick microcavities-1D PCs.

\section{Magnetophotonic crystals made by bonding}

Experiments on bonding a single-cavity MPCs with a structure of $\mathrm{SG} / \mathrm{S} /(\mathrm{T} / \mathrm{S})^{3} / \mathrm{M}-\mathrm{M} /(\mathrm{S} / \mathrm{T})^{3} / \mathrm{S} / \mathrm{SG}$, where $\mathrm{SG}$ was an SGGG $\left((\mathrm{GdCa})_{3}(\mathrm{GaMgZr})_{5} \mathrm{O}_{12}\right)$ substrate and $\mathrm{M}$ is a $\lambda / 4$-thick $\mathrm{Bi}$ :YIG layer, could not be done because of the weak resistance of $\mathrm{Bi}$ :YIG against $\mathrm{H}_{2} \mathrm{SO}_{4}$. The $\mathrm{Bi}$ :YIG layer peeled off, and the surface of the sample became rough. The root-mean-square roughness $R$ was greater than $1 \mathrm{~nm}$; it is known that for successful bonding, $R$ has to be less than $1 \mathrm{~nm}{ }^{14)}$. To avoid such a degradation of the garnet layer, the activation of the Bi:YIG surface was achieved by the ion beam milling. Surfaces of two fractions can be activated by ion beam etching and brought into contact in vacuum 14, 15, 16). Also, our experiments showed that Bi:YIG layers fabricated on synthetic fused quartz substrates had a number of micro cracks. This was caused by the thermal 


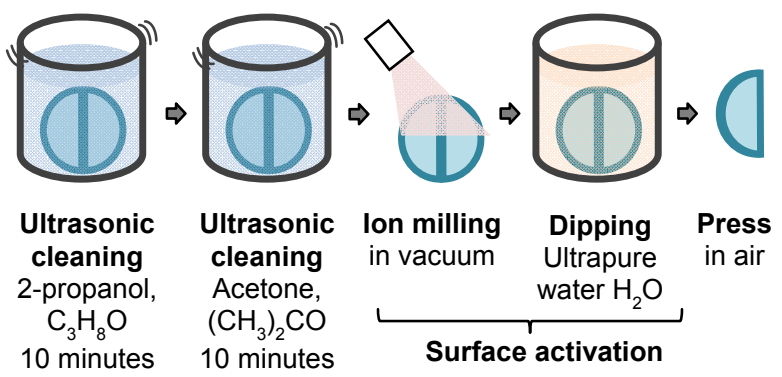

Fig. 5 Bonding procedure with surface activation by ion milling.

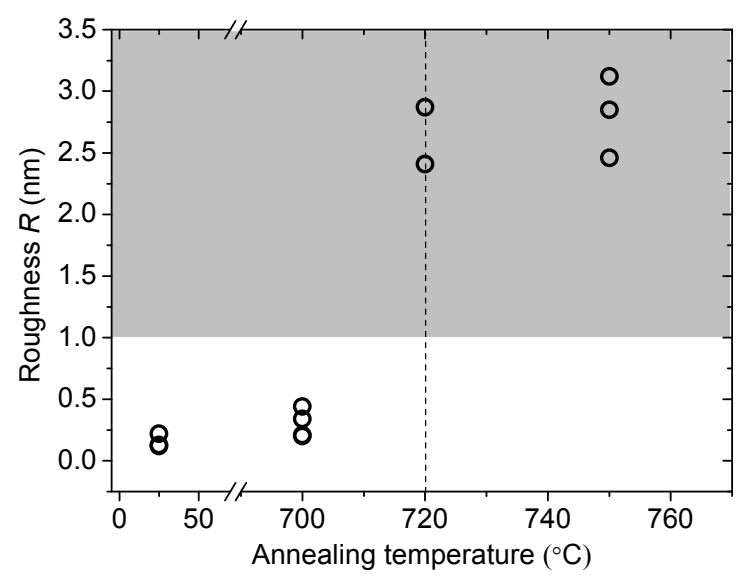

Fig. 6 Surface roughness of a $\mathrm{Q} /(\mathrm{T} / \mathrm{S})^{2}$ multilayer; the annealing time was 15 minutes in air. The dashed line shows a temperature of $\mathrm{Ta}_{2} \mathrm{O}_{5}$ crystallization. The gray zone shows the destructive condition for successful bonding ( $R$ $>1 \mathrm{~nm})$

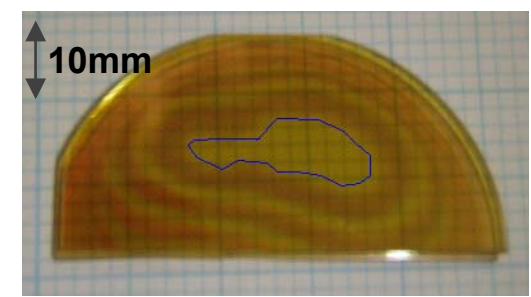

Fig. 7 Bonded single-cavity MPC with a structure of $\mathrm{SG} / \mathrm{S} /(\mathrm{T} / \mathrm{S})^{5} / \mathrm{M}-\mathrm{M} /(\mathrm{S} / \mathrm{T})^{5} / \mathrm{S} / \mathrm{SG}$ : the bonded area is encircled.

expansion between the substrate and the layer. Also, these cracks introduced additional surface roughness and light scattering. That is why, to avoid formation of cracks, we used of SGGG substrates with the coefficient of thermal expansion similar to that of Bi:YIG.

To crystallize Bi:YIG, the samples were annealed at $700{ }^{\circ} \mathrm{C}$ for 15 minutes. Annealing determined the surface roughness after crystallization: $R=0.2 \mathrm{~nm}$ (before) and $R=2.8 \mathrm{~nm}$ (after). Figure 5 illustrates the fabrication procedure. The cleaned samples were etched by the ion beam in a vacuum chamber. It is worth noting that $R>1.0 \mathrm{~nm}$ was also found for the surfaces of Bragg mirrors annealed at temperatures of $>720{ }^{\circ} \mathrm{C}$

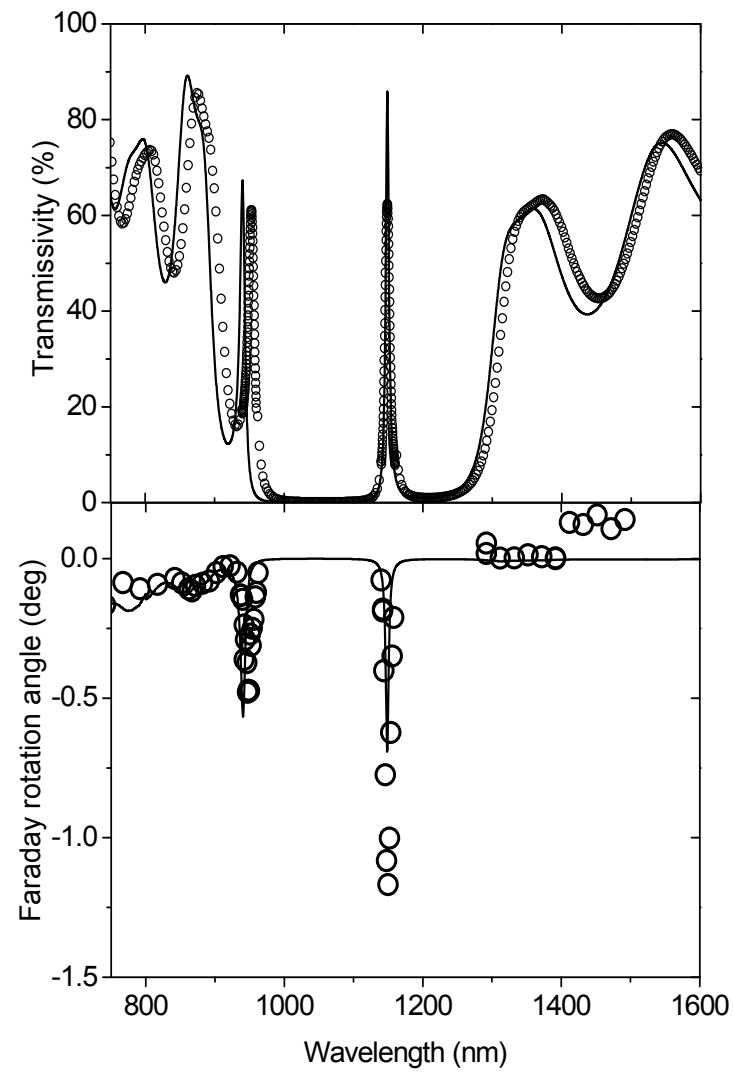

Fig. 8 Transmission and Faraday rotation spectra of the MPCs shown in Fig. 7 (experiment-symbol, calculation-line).

because of $\mathrm{Ta}_{2} \mathrm{O}_{5}$ crystallization (see Fig. 6).

Figure 7 shows a photograph of a bonded single-cavity MPC sample with a structure of $\mathrm{SG} / \mathrm{S} /(\mathrm{T} / \mathrm{S})^{5} / \mathrm{M}-\mathrm{M} /(\mathrm{S} / \mathrm{T})^{5} / \mathrm{S} / \mathrm{SG}$. One can see the bonded area marked by a curve in the center of the MPC; the surface area was $0.19 \mathrm{~cm}^{2}$. Note that the $\mathrm{SG} / \mathrm{S} /(\mathrm{T} / \mathrm{S})^{5 / \mathrm{M}}$ multilayer was annealed at $700{ }^{\circ} \mathrm{C}$ for 15 minutes, and the resultant roughness was $R=0.2 \mathrm{~nm}$. In this structure, the Bi:YIG layers were $285^{-n m}$ thick, and the Bragg mirrors were manufactured by Ito Kogaku Co. Ltd. Transmissivity and the Faraday rotation angle of the MPC discussed in Fig. 7 are presented in Fig. 8. One can see a resonant peak at $1149 \mathrm{~nm}$ within the stop band ranging from $1000 \mathrm{~nm}$ to $1300 \mathrm{~nm}$ and the corresponding enhancement of the Faraday rotation angle $\theta_{\mathrm{F}}=-1.17^{\circ}$. However, the intensity of the Fabri-Perot peak was significantly smaller than the calculated one. Taking into account that Bi:YIG has a negligible optical absorption in this spectral range, it is most likely that the annealing and bonding procedures destructively influenced on the optical response. Calculated consideration ${ }^{17)}$ shows that the deviation of the thickness for the bonding interface layer makes the destructive interference. In general, the experimental spectra were in the excellent agreement with the calculated responses. This means the activation process using ion milling is beneficial for the fabrication of the symmetrical MPC, and it inferred that the process is 

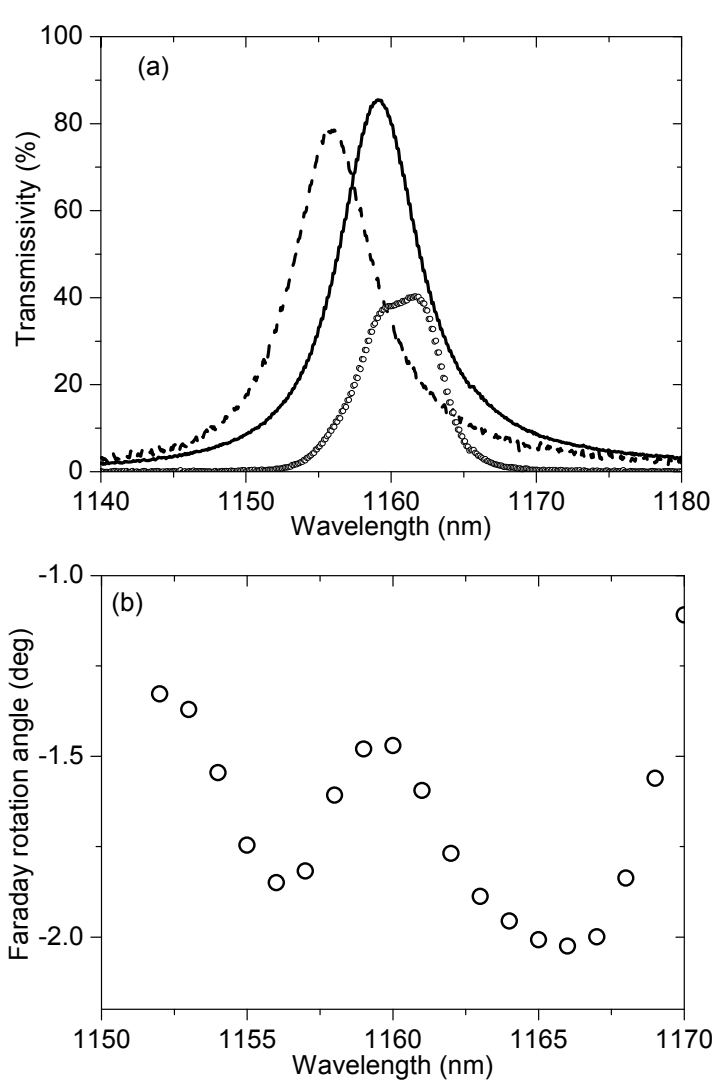

Fig. 9 (a) Transmission and (b) Faraday rotation angle spectra of a dual-cavity MPC (circles) with a structure of $\quad \mathrm{SG} / \mathrm{S} /(\mathrm{T} / \mathrm{S})^{5} / \mathrm{M} /(\mathrm{S} / \mathrm{T})^{5} / \mathrm{S}-\mathrm{S} /(\mathrm{T} / \mathrm{S})^{5} / \mathrm{M} /(\mathrm{S} / \mathrm{T})^{5} / \mathrm{S} / \mathrm{SG}$. Transmission spectra of the initial $\mathrm{SG} / \mathrm{S} /(\mathrm{T} / \mathrm{S})^{5} / \mathrm{M} /(\mathrm{S} / \mathrm{T})^{5} / \mathrm{S}$ single cavities (solid and dashed lines) are shown for comparison.

also beneficial for the fabrication of the dual-cavity MPC.

Fabrication approach discussed above was applied for forming dual-cavity MPCs. For this experiment, two single-cavity MPCs having structures of $\mathrm{SG} / \mathrm{S} /(\mathrm{T} / \mathrm{S})^{5} / \mathrm{M} /(\mathrm{S} / \mathrm{T})^{5} / \mathrm{S}$ were used; the roughness of the $\mathrm{Bi}$ :YIG surface after annealing was $R=0.95 \mathrm{~nm}$. Figure 9 shows experimental transmission and Faraday rotation angle spectra of the bonded dual-cavity MPCs and initial single-cavity MPCs (two before bonding samples). Double peaks were observed in the Faraday rotation spectrum of the dual-cavity MPCs, proving successful bonding. However, transmission spectra of the single cavities had $3-\mathrm{nm}$-detuned resonances in the vicinity of $\lambda=1160 \mathrm{~nm}$. This significantly decreased the intensity of the resonant peak and the magnitude of Faraday rotation. It should be noted that the dual-cavity MPCs degraded within a few hours and, finally, the initial single cavities detached. It is most likely that detaching happened because of roughness. The roughness $R=0.95 \mathrm{~nm}$ of dual-cavity MPC was larger than that of single-cavity $R=0.2 \mathrm{~nm}$. It is close to the destructive condition of bonding shown in Fig. 6 .

\section{Conclusion}

We have demonstrated that the bonding method is applicable for fabrication of single- and dual-cavity MPCs. Spectra of the bonded MPCs revealed Fabri-Perot peaks due to resonant light propagation and related enhancement of the Faraday rotation. Destructive influence of garnet annealing on optical responses of dual cavities have been found. This work showed that for successful bonding of magnetic garnet-based multilayers further development of bonding procedures is necessary.

Acknowledgements This work was supported in part by Research Fellowships of Japan Society for the Promotion of Science (JSPS) for Young Scientists Grant No. 21-7394 and Global COE Program "Frontiers of Intelligent Sensing" from the Ministry of Education, Culture, Sports, Science and Technology, Japan.

\section{References}

1) M. Inoue, R. Fujikawa, A. V. Baryshev, A. Khanikaev, P. B. Lim, H. Uchida, O. Aktsipetrov, A. Fedyanin, T. Murzina, and A. Granovsky: J. Phys. D, 39, R151 (2006).

2) A. B. Khanikaev, A. V. Baryshev, M. Inoue, A. B. Granovsky, and A. P. Vinogradov: Phys. Rev. B, 72, 035123 (2005).

3) A. M. Merzlikin, and A. P. Vinogradov: Opt. Comm., 259, 700 (2006).

4) H. Kato, and M. Inoue: J. Appl. Phys., 91, 7017 (2002).

5) M. J. Steel, M. Levy, and R. M. Osgood, Jr.: IEEE Photon. Technol. Lett., 12, 1171 (2000).

6) S. Fan, and Z. Wang: J. Magn. Soc. Jpn., 30, 641 (2006).

7) H. Kato, T. Matsushita, A. Takayama, M. Egawa, K. Nishimura, and M. Inoue: Opt. Comm., 219, 271 (2003).

8) H. Kato, T. Matsushita, A. Takayama, M. Egawa, K. Nishimura, and M. Inoue: J. Magn. Magn. Mater., 272-276, e1327 (2004).

9) H. Takagi, A. Tsuzuki, K. Iwasaki, Y. Suzuki, T. Imura, H. Umezawa, H. Uchida, K. H. Shin, and M. Inoue: J. Magn. Soc. Jpn., 30, 581 (2006).

10) H. Takagi, J. Kim, K. H. Chung, S. Mito, H. Umezawa, and M. Inoue: J. Magn. Soc. Jpn., 33, 525 (2009).

11) M. J. Steel, M. Levy, and J. R. M. Osgood: J. Lightwave Technol., 18, 1297 (2000).

12) T. Goto, A. V. Baryshev, K. Tobinaga, and M. Inoue: J. Appl. Phys., 107, 09A946 (2010).

13) S. Wittekoek, T. J. A. Popma, J. M. Robertson, and P. F. Bongers: Phys. Rev. B, 12, 2777 (1975).

14) H. Takagi: J. J. Weld. Soc., 69, 156 (2000).

15) T. Oda, T. Abe, and I. Kusunoki: J. Vac. Soc. Jpn., 49, 310 (2006).

16) S. Suehara, M. R. Howlader, and T. Suga: IEICE Trans. Electron., 88, 920 (2005).

17) T. Goto, Y. Haga, A. V. Baryshev, and M. Inoue: J. Phys.: Conf. Ser., 266, 012009 (2011). 